possible in all manipulations; and (5) counteracting shock by all means possible. Strychnine and brandy were administered before and after the operation. Gastric lavage was employed before and after operation in cases where vomiting was present. Large quantities of saline solution were administered after operation per rectum every four hours, sometimes with brandy added. Nothing was allowed by the mouth for the first 24 hours after operation except a teaspoonful of albumin water every half hour, and not this if any sickness was present. One-sixth of a grain of calomel was administered at once after operation and one-sixth of a grain every fourth hour until one and a half grains had been given. Turpentine enemata were used with the rectal tube passed high up to relieve flatus and distension. And, lastly, but by no means to my mind the least important, the patients were placed in the Fowler position by means of a bolster beneath the knees and a back-rest, the bolster being retained in position by two bandages tied to the bed head on either side.

I think there are other points of great importance in influencing the result of operative treatment. Firstly, rapidity combined with gentleness in manipulation and thoroughness of operation. Secondly, the administration of the anæsthetic. If the patient has a terror of the operation or is excessively nervous the initial administration of ethyl chloride, I believe, is extremely useful, as it renders the patient anæsthetic in about 30 seconds and allows the exhibition of ether or chloroform to follow it at once without difficulty. It also shortens the duration of the operation in that often the surgeon, if he sees that he has everything prepared, may make the incision under the ethyl-chloride anæsthesia and may have time to open the peritoneum before its effects have completely passed off. This allows of the operation being carried through with the minimum of ether or chloroform, it being only needful to push it a little towards the end if sutures are to be introduced or another incision made for drainage. I consider the limitation of the anæesthetic to the minimum necessary as of extreme importance in these cases. It is also of great importance to keep the patient warm during operation, and to this end a hot-water tank on the operating table is very valuable, and also I employ a jacket for the patient's body with sleeves out of which there is no opening, a small slit being provided at the wrist to enable the pulse to be felt. I also have leggings with socks all in one which pull up to the top of the thigh. These are made of thick woollen blanket and, I find, keep the patient warm. As little of the surface of the body should be exposed as possible.

Lancaster.

\section{THE TREATMENT OF CHRONIC SUPPURA- TION OF THE MIDDLE EAR WITHOUT THE REMOVAL OF THE DRUM MEMBRANE AND OSSICLES.}

BY CHARLES J. HEATH, F.R.C.S. ENG.,

SURGEON TO THE THROAT HOSPITAL, GOLDEN-SQUARE, LONDON.

THERE should no longer be any doubt of the fact that chronic suppuration of the middle ear, if promptly treated, is usually curable, with restoration of hearing and with healing of the perforated drum membrane. It appears also that practically every man or woman who suffers from chronic suppuration of the middle ear has disease of the mastoid antrum, and although occasionally the discharge may cease with or without treatment on account of a favourable anatomical formation of the antrum and aditus, this fact does not invalidate the contention that while there is chronic suppuration there is disease of the mastoid antrum. These statements are founded on the experiences of over 500 mastoid operations performed during the various stages of this disease, and in all those patients not in one instance was the mastoid antrum in a condition of health.

Since the publication of a previous paper on this subject ${ }^{1}$ many surgeons have seen my patients and operations at the Throat Hospital and have adopted the procedure advocated in a large number of cases with great success. The operation affords no great difficulties, nor is it accompanied by much danger, for after years of gradual diminution mortality has now disappeared among those patients submitted to opera. tion before dangerous symptoms have developed.

The patients referred to in my previous paper were admitted to the Golden-square Hospital because they were serious cases of chronic suppuration in the middle ear; they all had perforation in some part of the tense portion of the drum membrane and the aural disease varied in duration from three months to 18 years. Some had polypus in the meatus, others granulations in the tympanum, one had a discharging sinus behind the ear, all had deafness and foetid discharge, and some in whom the disease had become acute were in great danger from extensive exposure of the dura mater of the middle brain and the lateral sinus in the acute mastoid abscess. Yet such severe cases as these were shown to be capable of complete recovery, with cessation of discharge, healing of the perforation, and perfect restoration of hearing. These ten patients were exhibited at a meeting of the British Laryngological and Otological Association ${ }^{2}$ and the result of the discussion was greatly in favour of the new operation. One or two who took part in the discussion expressed their doubts as to the new method being adequate to deal with "attic" disease (so-called). In my reply, the belief was expressed that by operating in time it wonld be possible to prevent the "attic" from being involved and that recently, when operating fairly early on three patients with "attic" disease, serious disease of the mastoid antrum had been found in all of them, and probably the "attic" disease would be recovered from when the diseased cavity of the antrum was eradicated. These expectations regarding the possible cure of "attic" disease were so far fulfilled that at the next meeting of the association, a month later, two of those three "attic" patients were exhibited completely recovered from the disease, with the discharge ceased, with the perforation in the membrane healed, and with much hearing restored. The third patient had been removed from the hospital before recovery because she developed an infectious disease which demanded her removal to another institution.

Not only are the ordinary forms of chronic suppuration of the middle-ear amenable to the new treatment, but it is equally effective in the attic form also. In October and November last some of the rarer and more complicated forms of suppuration came under observation and operation, such as "attic" disease (so-called) with perforation of Shrapnell's membrane and chronic suppuration associated with facial paralysis. Particulars of these cases are recorded below and, in addition, details of a most interesting and instructive case in which the operation of ossiculectomy had been performed 12 years previously for the purpose of attempting to cure chronic suppuration in the ear.

The following five cases were exhibited at the meeting of the British Laryngological and Otological Association on Dec. 7th, 1906 :-

CaSE 1. Attic disease (so-called).-The patient, aged 24 years, was admitted to the Throat Hospital on Sept. $21 \mathrm{st}$, 1906, complaining of pain in the right ear, also of a feeling of giddiness even when lying down. There had been discharge for six months from this ear, in which there was a perforation of Shrapnell's menabrane. She could hear the watch at a distance of one inch. The Eustachian tube was free but not communicating with the perforation, there being some obstruction to the passage of air through the tympanum, and in order to attempt to save the hearing of this, her only useful ear, operation was decided upon and was performed on Oct. 11th. The conservative operation was adopted, as described in THE LANCET of August 1lth 1906 , p. 353.

There was pus in the antrum and that cavity was draining through a perforation in Shrapnell's membrane. The probe was passed completely through the posterior part of the "attic" from the perforation in the membrane to the aditus; air was then blown through. Next, having obstructed the perforation with a wet woollen mop, an attempt was made to force air forwards through the tympanic cavity, from was made to force air forwards through the tympanic cavity, from the aditus to the anterior part of the tympanum near the Eustachian the, and to distend the tympanum, but without success ; it escaped through the perforation in spite of the occlusion of that opening by means of the wet woollen mop firmly held. Next, by incision, an and made in the anterior inferior quadrant of the Dhich had been prepared for the purpose opening, and an attempt was made of th the obstruction in the tympanic cavity was unyielding. No further the obstrustion in the tympanic cavity

In this patient a small part of the bridge above the perforation was In this patient a small part of the bridge above the perforation was remer cases in order to examine the atic blow alr through the perforation by the obstinate tympanic obstruction had been spontaneously removed; in the mene 
be heard at a distance of one inch only; at the present time it can be heard at five inches. This is a most useful ear; it is her only available one, and fortunately it is now safer than if the ear had never been diseased, for the mastoid antrum (the danger zone) has been removed;
also all discharge has ceased and the perforation in Shrapnell's also all discharge has

membrane "Attic" disease.-The patient was first seen in July, 1906, CASE 2. "Attic" disease. The patient was first seen in July, 1906,
with a history of discharge from the left ear lasting two months, follow with a history of discharge from the left ear lasting two months, follow
ing one week of pain. There were giddiness and slight headache. The wateh was not heard on contact. Air cuuld not be blown through the wateh was not heard on contact. Air could not be blown through the perforation by Valsalva's method, though the Eustachian tube was after its removal it was possible to see that the perforation was in Shrapnell's membrane. Spirit drops were ordered to be instilled several times a day.

On a subsequent visit a small recurrent, polypus was removed and it was found that the otorrhcea had increased. In these circumstances operation was recommended and the conservative one was performed on Oct. 25th. Cheesy pus and a few granulations were found in the antrum and pus in some mastoid cells communicating with the antrum. On opening the cartilaginous meatus as usual towards the conclusion of the operation the membrane was fully exposed and then were removed some granulations from within the attic; a probe was passed through the perforation and the attic into the aditus, air was then blown through. On looking into the posterior attic granulations could be seen.

The experiences gained by the operation on Case 1 did not encourage the attempt to force the tympanic obstruction. Four days after the operation this patient also could blow air through the perforation, which indicated that the tympanic barrier had disappeared. Before the operation the watch could not be heard when in contact with the ear; at the present time it is heard 11 inches away. His recovery was uneventful : the perforation healed and all discharge ceased.

CASE 3. "Attic" disease.-The patient was admitted to the hospital on Nov. 2nd, 1906, with a history of fonr months' discharge from the left ear, tinnitus, and pain over the mastoid. The watch was heard only when in contact with the ear. There was a greyish granulation only when in contact with the ear. There was a greyish granulation
of about the size of a pea apparently growing through the roof of the of about the size of a pea apparently growing through the roof of the
meatus but in reality through the upper part of Shrapnell's membrane. Air could not be blown through the perforation from the nose, although the Fustachian tube was clear.

The conservative operation was performed on Nov. 16th. The aditus contained thick pus, and on probing from behind through the attic to the perforation after removal of granulations some white material was thrust out through the perforation, which on examination proved to be a flake of glistening epithelium of about the size of the section of a
lead pencil. The probing was continued and two or three similar flakes were removed in the same way. Then air was blown through and all desquamated epithelium was cleared away. This patient progressed satisfactorily and in a few days could blow air through the perforation by Valsalva's method, but developing an infectious disease she was removed. This was a most interesting case and her departure was disappointing.

CASE 4. Facial paralysis.-The patient was admitted to the hospital on Nov. 12th, 1906, with a history of nine days' facial paralysis and a discharge from the right ear of 27 years' duration. The paralysis was as marked as in any case in mv experience, and the patient's demanded the use of pad and bandage to keep the lid down and thu protect the cornea.

The conservative operation was performed on Nov. 15th. The mastoid antrum contained granulations and pus. Air and lotion were passed through the tympanum from the aditus and forced out through the perforation. There were no apparent granulations, even after 27 years' suppuration. This patient went home on Dec. 2nd. The facial years' suppuration. This patient went home on Dec. 2nd. The facial paralysis had disappeared, the discharge had ceased, and she could shut the eye of the affected side as strongly as the other. Before the opera-
tion she heard the watch at two and a half inches; she now hears it tion she heard the watch at two and a half inches; she now hear

CASE 5 . An old ossiculectomy case.-The patient was admitted to the CASE 5. An old Ossiculectomy case. - The patient was admitted to the
hospital on Nov. 5 th for operation on the left ear, after two years treatment as an out-patient. There had been otorrhcea for 14 years but never any pain. There was a cicatricial band on the inner wall of the tympanum and below it a carious patch. The whole tympanum
was wet with foetid pus. She could hear the watch at a distance of was wet with foetid pus. She could hear the watch at a distance of
two inches in that ear and in the other at 14 inches. An operation had been performed at another hospital, probably ossiculectomy and curetting.

The conservative operation was performed on this patient also. The antrum was large and full of granulations wet with pus. The aditus

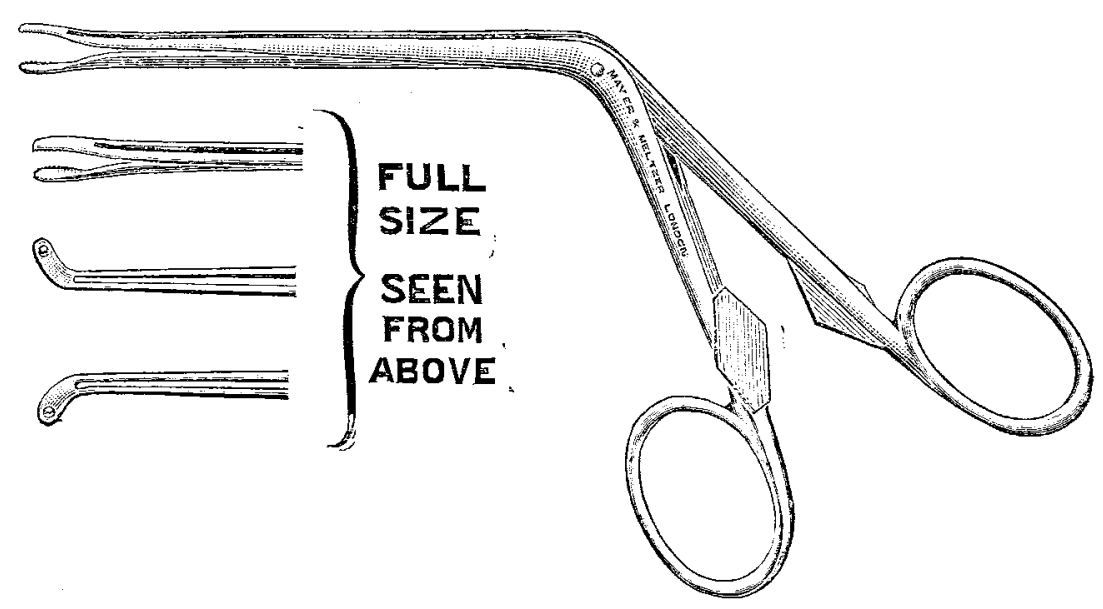

Forceps for removing granulations, \&c., from the tympanum or attic. The full-sized ends show the three patterns which are in use-the straight forceps above, tho
those bent to the left below. contained granulations. The removal of the pyogenic membrane ining the antrum and aditus and therefore the cessation of supply of pus to the tympanum permitted the latter, although it was in no way treated, to make a satisfactory and rapid recovery of course, not a perfect recovery, as the drum membrane and ossicles had been taken away by the operation of ossiculectomy 12 years before. The caries rapidly healed and all discharge ceased. The patient was in the hos pital three weeks and on leaving could bear the watch at four and If inches, twice as far as before the operation.

The foregoing notes on these five cases provide matters demanding serious consideration. To take the so-called "attic" cases first, it may be observed that all had disease of the mastoid antrum although the aural discharge was not of long duration nor were there any mastoid symptoms. All had such swelling of the tympanic mucous membrane as to prevent the passage of air and discharge from the antrum or perforation to the Eustachian tube or vice versâ. In all that firm obstruction spontaneously disappeared within a week of the operation-that is, as soon as the irritation of the tympanum, arising from the presence of pus from the antrum, had been eliminated by the operative proceedingsand none had "attic" disease, so understood, unless the accidental ingrowth of epithelium in one of them could be so called. This ingrowth of epithelium in time might have spread and its products have accumulated anywhere within the tympanum, but more especially in the posterior " attic" portion, for the rest of the tympano-attic cavity was obstructed by the barrier of swollen mucous membrane referred to above or by granulations, therefore the accumulation taking place in the direction of least resistance would naturally occur in the " attic."

How these facts concerning " attic" disease contrast with what has so long been taught about it! And this information is the result of close observation of the living and conld not possibly have been demonstrated post mortem; each of these persons suffered from disease of the mastoid antrum affecting secondarily both the attic and tympanum, and in one patient the conditions leading to cholesteatoma were present. The locality in which the perforation occurred appeared to depend on the amount of swelling in the tympanum, which by protecting the rest of the membrane insured that perforation should take place in Shrapnell's membrane; it was the only part exposed.

In the two cases which remained under observation the discharge ceased, the perforations healed, and the hearing greatly improved; also the vertigo and pain disappeared, implying a termination of the disease, due, not to treatment of the tympanum but to the elimination of the mastoid antrum and its putrid discharge which had been irritating the tympanum. Although the giddiness was due to tympanic conditions, treatment of the tympanum, except destructive operation, would not have removed it. The main disease was not in the tympanum or attic at all but in the mastoid antrum-it was mastoid disease, and thus it should be named. The patient in Case 1 , in whom air was blown through an artificial opening, showed that the obstruction to the passage of air through the tympanum was unyielding and that there fore the apposition of the swollen mucous membrane of the inner tympanic wall to the drum membrane must have been an accurate moulding in order to prevent the passage of air under such a great pressure as was used in the attempt to force a passage. Cases 1 and 2 both complained of giddiness, which
can be accounted for by the external pressure exercised upon the incus, by means of the swelling of the mucous membrane of the tympanic inner wall, which, pushing out the lower part of that bone until further progress was resisted by its anchorage to the stapes, pulled upon the stirrup and by dragging on the ligaments tended to diminish the pressure in the labyrinth. This was enough not only to account for the vertigo but might even account for the pain. Tympanic obstruction of a less degree is a very common condition in chronic suppuration and swelling of this kind combined with the amount of bloodsupply form the chief factors in deciding where a pertoration of the drum membrane shall take place.

The patient in Case 2, with giddiness, pain, and nerve deafness, might have been suspected of having labyrinthine implication, but the symptoms were probably the result of alteration of labyrinthine tension due to tympanic swelling as explained above. The labyrinth is far more rarely involved in this 
country than abroad, if statistics are to be considered reliable.

The hearing of these two patients considerably improved after their recovery, but it does not compare favourably with the cases already recorded in TIE LANCET and with many other cases treated since, and the reason is not far to seek, for the tension in, and therefore the sensibility of, the labyrinth was altered by the long-continued stretching of the stapedial ligaments; also, the ossicular chain of bones, and their intervening joints, and the vibrating: tympanic membrane itself had all suffered by the long-continued stretching and pressure to which they were subjected by the outward swelling of the mucous membrane of the tympanic inner wall. Even better results regarding the hearing might therefore havie been expected had they been operated upon even earlier, before the tympanic swelling had damaged the labyrinth and the ossicular chain and attachments. (The hearing of these two patients is slowly and steadily improving.) Again, the whole week of pain suffered by Oase 2 before the perforation occurred suggests the gradual accumulation of the drainage from the antrum which appears to secrete but little, and contrasts therefore with the abundant discharge observed when the tympanum is much affected.

Case 3 exbibited conditions indicating the commencement of cholesteatoma, and in this patient it appears to have been due to the implanting of the squamous epithelium of the outer surface of the drum membrane upon the granulation which happened to be in close contact with it. The squamous epithelium was probably first implanted upon the protruding granulation, which being large and coming like a button through a buttonhole might have been tightly nipped round its neck by the edge of the perforation and possibly excoriated there, and once the epitheliam from the outer edge of the drum membrane became implanted it might grow and travel over the surface of the granulation, along its stem, and thus inwards through the perforation, until it reached the general mucous membrane of the tympanic attic of which the granulation was a part, and there the effete epithelium becoming thrown off would naturally accumulate and lead to cholesteatoma; also the squamous epithelium might continue to extend over the mucous surface of the cavity. The grey-white appearance of the granulation itself might indeed have indicated a covering of squamous epithelium, but it was unfortunately not microscopically examined.

Observations such as these may assist in settling the disputed origin of cholesteatoma and should it altimately be generally concluded that this is the way in which it usually originates valuable hints are obtained as to what surgical or other treatment is desirable in order to prevent such a calamity.

From experiences of these cases there appears much difficulty in recognising any disease as peculiar to the attic as well as any peculiar to the tympanum. That such a division seems arbitrary, as the tympanum, and its upper part the attic, together form one tiny cavity; it is an attempt at a distinction without a difference, for granulations or caries may be present in either locality or in both, as in the cases recorded above, the disease merely being modified by the anatomy of the part. Further, that whether the pathological changes are apparently more in the tympanum or more in the attic, the conditions are secondary to disease of the mastoid antrum. Again, the discharge from the antrum which causes the attic or tympanic disease has to pass through the attic in every case to reach the perforation wherever it may happen to be, whether in the tympanic or attic portion of the drum membrane, and to call this disease by the name "tympanic" or "attic" because of the situation of the perforation, high or low, seems devoid of all reason. Moreover, practically all these cases are curable by the same operation if dealt with at the proper time. The following conclusion was therefore ultimately reached-namely, that however diverse the conditions in the ears of different persons may appear to be in the late stages of suppuration, they all appear to fall into one category in the early stages-that is, disease in the mastoid antrum-for it can now be demonstrated that the same principle governs them all, and therefore the same treatment may be expected to be effective in all if carried out at the proper time.

The above cases emphasise the importance of an early operation when the disease settles down into a condition of chronic suppuration. The majority of cases of acnte suppuration, however, recover completely; they do not become chronic.

The use of the Politzer bag is especially indicated after operations upon these attic cases, for the ossicles and joints have been pressed upon by swelling, and their loosening and shaking up and the breaking of their adhesions are as necessary here as in any other part of the body after somewhat similar conditions of strain and subsequently an enforced rest.

In Case 4 (with a history of suppuration for 27 years and of facial paralysis for nine days at the date of admission) there can be no reasonable doubt that the cause of the paralysis was the onset of caries, as anything short of that would probably not have affected the bony aqueduct and the nerve after such a long period of disease. This case demon. strated that such a serious disease as caries of the tympanum can be recovered from even without the removal of the drum membrane, as is done in the radical operation, if the cavity is freed from the irritating effects of putrid discharge from the mastoid antrum by a less destructive method, and as there was no apparent protective swelling of the tympanic mucous membrane in this patient there was greater liability to caries of the walls of the cavity as the result of the irritation from the discharge from the antrum.

In Case 5 an operation was performed 12 years ago on the patient through the meatus, probably ossiculectomy, with the hope of curing chronic suppurative catarrh. This case illustrates the fact that ossiculectomy was not to be relied upon to stop otorrhcea, but was in fact fatile, for it did not reach the dangerous disease in the mastoid antrum, and thus make the life of the patient safe in spite of the sacrifice of all possibility of restoring the hearing, and even if ossiculectomy would cure the mastoid disease the fact of its destroying the hearing should prevent its adoption. This case shows also that even without the presence of the drum membrane caries can be produced on the inner wall of the tympanum and perpetuated there by discharges draining down from the mastoid antrum, and that it can be recovered from when that discharge is arrested. There can be no doubt as to the presence of caries in this case, for bare bone could be felt by the probe and its situation proved by observation prior to the operation, but more fully demonstrated when the meatus had been enlarged, as is the rule in this method of operation.

To take the facial paralysis and ossiculectomy cases together. In each there was caries in the tympanum; in the first it appeared after 27 years of suppuration and in the second it was observed after disease of 14 years' duration. In both it disappeared when the discharge was arrested by operation on the mastoid antrum, the fountain-head of the suppuration which caused the caries, and not by operating on the seat of the caries in the tympanum. These facts, it will be observed, are in direct opposition to the teaching hitherto in vogue.

Caries is rare; so are the results of that disease such as facial paralysis and labyrinthine suppuration, yet suppuration is extremely common, and if caries was the cause of it, it should always be present. It does not need the presence of caries to cause perpetuation of suppuration, while the mastoid antrum is the factory of fotid pus, for which the tympanum has to act as the drain and is thereby constantly irritated and perpetually discharging. Caries is not, as has been hitherto taught, the cause of the persistence of suppuration, but according to the investigations noted above it is the effect of that persistence.

Further, and a most important point common to these five cases, serious diseases of the mastoid antram were found in all of them, even in those of but short duration-a matter that could only be demonstrated by an early operation such as was done in some of them; and this fact again emphasises the contention that practically all cases which do not recover after acute catarrh and relapse into a chronic suppuration have disease in the mastoid antrum. As remarked before, for practical purposes there is no exception to this rule, and the fact that some cases do recover on account of exceptionally favourable conditions of the aditus and antrum (not to be relied upon nor to be diagnosed) does not invalidate the contention that while there is chronic suppuration going on there is disease in the mastoid antrum. The results of these and other operations appear to show that those ears are the healthiest and most vigorous in which the tympanic swelling and granulations are most abundant. 
Cases which are devoid of apparent granulations are slower in recovering after operation, and by being less protected are more liable to the penetration of disease into the tympanic walls leading to caries, a far more serious condition, as in the facial paralysis case. Many other pathological matters noticed in this investigation are in entire conflict with the teaching hitherto accepted, and if the old pathology were right the gratifying results of this new treatment at the hands of many surgeons would be impossible; it is however founded, and fortunately so, upon a new aspect of pathology of this disease.

Some surgeons have lately informed me that they are operating by this new method on patients whom they have had under observation for years, but dared not operate by the radical operation as it might destroy the hearing of the only useful ear (such conditions prevailed in Case 1). This operation might be called the conservative operation, because it is designed to save what is essential to perfect hearing; but the name is a small matter as the evolution of the operation was a certainty once the true pathology was known. What, however, was most important was the recognition of the fact that the pathology hitherto taught was not to be relied on, and the duty of acquiring a more accurate knowledge of the nature of this serious disease has demanded years of the closest clinical study, for as long as the old pathology dominated the surgery of this disease no advance was possible; as long as the tympanum was considered the root of the disease so long would the disastrous results of the radical operation and ossiculectomy be perpetuated.

Many questions as to the scope of this operation have reached me from surgeons in this country and abroad; it is not a simple opening up of the antrum and a clearing of that cavity and the aditus followed by drainage, as in Schwartze's operation, which caused so many disappointments and led to the institution of the radical operation, which, on the other hand, went much too far. In the new operation not only the posterior superior part of the meatus is removed, as in some other operations, but the antrum and aditus are practically eliminated, as weil as all disease associated with those cavities. The whole posterior wall of the bony meatus and even its arched floor must also be removed, and this latter proceeding is followed in no other operation, but it must be done if that full access to the tympanum which is necessary both during and after the operation is to be assured. This is essential not only for the safe and thorough removal of granulations, polypi, \&c., at the operation, but especially for the dressings during the after-treatment, for without the removal of the floor it is impossible to see the whole of the mem. brane; moreover, a pocket would be left between the arched floor and the membrane where discharge might accumulate.

Already over 100 of these operations have been performed in the country for chronic suppuration, and it is also being practised in America. Between 20 and 30 have taken place at my hands; two surgeons of my acquaintance have, together, and in a shorter period, performed a similar number, and one of them, a resident in a northern city, is so satisfied with his results that some months ago he had adopted this method in 12 out of 15 mastoid operations and has exhibited some of his patients at the local branch of the British Medical Association.

It is not claimed that under all diseased conditions of the tympanum this elimination of the antrum and aditus is to be expected to restore the ear to health, for the drum mem brane may be extensively destroyed or completely gone; the ossicles, too, may have necrosed and disappeared, as sometimes happens in scarlet fever and other acute diseases, but this does not occur in chronic disease. For such cases the radical operation will still be required, yet they form a small proportion and will rapidly diminish, as the treatment of this disease is more promptly undertaken on the lines recommended herein. The surgeon's aim should be the restoration of a perfect ear. The aim, however, of the radical operation and the hope of ossiculectomy is to get the discharge stopped with a certainty of a defective ear.

It is as much the duty of the aural surgeon to operate in time to save the hearing as it is that of the ophthalmic surgeon to operate in time in chronic glaucoma to save the sight.

Cavendish-place, $\mathrm{w}$.

\section{RECENT DEVELOPMENTS IN THE THERA- PEUTICAL APPLICATIONS OF ARSENIC.}

Bx J. SNOWMAN, M.D. BRUx., M.R.C.P. LoND.

MODERN advances in the progress of pharmacentical science are not confined to the invention of highly complex and synthetic organic preparations. Research must necessarily be prosecuted along this line because it has already produced remedies of permanent value to our daily pharmacopoia. But though investigation may mainly proceed in this direction, it will be occasionally worth while to devote some attention to our old-fashioned and time-honoured drugs. This attention will often be rewarded by the discovery that neither all their therapeutical nor their pharmaceutical applications have been exhausted. Some of the recent studies in arsenic exemplify this contention in a striking degree and a review of the present position of this drug in the treatment of disease may therefore be both opportune and profitable.

Arsenic must always command the interest of the clinician because its toxicology is so reminiscent of pathological processes. It is often instructive to study the action of drugs from the point of view of their influence either on structure or on function. The pharmacology of opium, strychnine, and digitalis, for instance, mainly concerns function; while potassium iodide, phosphorus, and arsenic may be regarded as drugs the value of which chiefly deperds upon the circumstance that they produce structural alterations in the tissues. If the alteration tends to restore a pathological condition of a tissue to its normal state the drug will be credited with a specific curative action. Our control of pharmacological action is, however, too imperfect to enable us to arrange our employment of remedies in such a manner as to guarantee that structures of morbid anatomy will revert into those of health. The group of drugs formerly known as alteratives possess the property of modifying the nutritive processes in the body. It is obvious that this effect involves definite chemical changes in cell constitution, and advanced changes of this character inevitably modify structure. The great value of alteratives in therapeutics is that they often change pathological processes brought about by disease into physiological processes. Their failure frequently arises from the circumstance that they introduce into the body their own toxic pathological processes. This is exceedingly well exemplified by arsenic, which is able to produce a mimicry of several diseases, much to the discomfiture of diagnosticians. In the nervous system we may have peripheral neuritis with rapid muscular atrophy, or definite myelitis. The intestinal canal may present appearances closely resembling cholera, with grannlar changes proceeding to fatty degeneration in the epithelial lining, the interstitial tissue and Peyer's patches being invaded by large round g ranulated cells. The liver may be affected in such a manner as to suggest a diagnosis of acute yellow atrophy. The skin may be the site of lesions the number of which is almost legion. Every anatomical manifestation known to dermatology from a slight erythema to a bulla is represented. The most characteristic appearances, however, are pigmentation, herpes zoster, and hyperkeratosis of the palms and soles. Hutchinson maintains that these thickenings may develop into true epitheliomatous growths.

The sphere of action of arsenic is obviously very extensive when it is allowed to act on the general healthy organism. Its danger lies in its tendency to cause fatty infiltration and degeneration, and its therapentical applications are accordingly very much hampered, though practical medicine still indicates a considerable field for its employment. Happily, recent investigations are indicating how the drug may be used so as to obtain its therapeutical effects with a considerably diminished risk of incurring its toxic consequences. It is probable that the organism itself provides a defence against arsenical poisoning, in a manner similar to the production of an antitoxin in the case of certain bacterial poisons. It is found that subcutaneous injection of arsenious acid into rabbits in large but not fatal doses followed by a prolonged leucocytosis. This increase in polymorphonuclear leucocytes, it should be added, is preceded by a temporary decrease in their number. The point, however, is that after the increase has occurred each leucocyte contains arsenic. If a fatal dose is injected no 